\title{
Effects of Lesions in the Medial Prefrontal Cortex on the Activity of Midbrain Dopamine Neurons
}

Seong S. Shim, M.D., Ph.D., Benjamin S. Bunney, M.D., and Wei-Xing Shi, Ph.D.

To determine whether lesions in the prefrontal cortex (PFC) alter the activity of midbrain dopamine (DA) neurons, single unit recordings were made from $D A$ neurons in control and lesioned rats. PFC lesions, obtained by local injection of ibotenic acid into the medial PFC, had no effect on either firing rate or bursting activity of $D A$ neurons in the ventral tegmental area (VTA). However, the number of spontaneously active DA neurons in the VTA was

KEY WORDS: Prefrontal cortex; Ventral tegmental area; Substantia nigra; Dopamine; Ibotenic acid; Single unit recording

Lesions in the prefrontal cortex (PFC) have been suggested to lead to an overactivity of dopamine (DA) in subcortical structures. Such an interaction between cortical and subcortical DA systems may play a critical role in the development of psychotic symptoms in schizophrenia (see reviews by Weinberger 1987; Grace 1991; Deutch 1992). Whereas studies, in general, support this suggestion, the mechanism through which the PFC interacts with subcortical DA systems remains unclear. One possibility is that glutamate released from cortical

From the Departments of Psychiatry (SSS, BSB, W-XS) and Pharmacology (BSB), Yale University School of Medicine, New Haven, Connecticut.

Address correspondence to: Wei-Xing Shi, Ph.D., Department of Psychiatry, Yale University School of Medicine, 333 Cedar Street, SHM B272, New Haven, CT 06510.

Received July 25, 1995; revised January 19, 1996; accepted January 30, 1996. significantly decreased. In the substantia nigra (SN), the same lesions increased the firing rate and had no effect on either the bursting activity of the number of active DA cells. These results suggest that PFC lesions alter the activity of DA neurons. However, VTA and SN DA neurons may respond differently to PFC lesions. (C) 1996 American College of Neuropsychopharmacology [Neuropsychopharmacology 15:437-441, 1996]

terminals acts directly on DA terminals in DA target areas to cause DA release. Consistent with this suggestion are the findings that glutamate and DA terminals in the striatal complex are often in close proximity to each other (e.g., Bouyer et al. 1984; Sesack and Pickel 1992) and glutamate applied locally in the striatum in vivo or in striatal slices induces a significant increase in DA release (e.g., Marien et al. 1983; Jin and Fredholm 1994; Ohta et al. 1994). However, as PFC projection neurons may also make direct synaptic contacts with DA neurons located in the midbrain (Sesack and Pickel 1992) and regulate the firing activity of DA neurons (Thierry et al 1979; Gariano and Groves 1988; Murase et al 1993), it is possible that PFC lesions lead to an alteration of the activity of midbrain DA neurons, thereby influencing the subcortical DA transmission. Consistent with this suggestion, Taber et al. (1995) recently showed that application of glutamate antagonists to the VTA blocks DA release in the nucleus accumbens induced by activation of the PFC. In this study, we used single unit recording techniques to record directly from DA neurons in the midbrain to determine whether their activity is altered by lesioning the PFC. 


\section{MATERIALS AND METHODS}

Male Sprague-Dawley rats weighing between 200 and $320 \mathrm{~g}$ were divided into three groups: lesioned $(n=$ $12)$, sham-lesioned $(n=9)$, and nonlesioned $(n=16)$. In the first two groups, rats were first anesthetized with chloral hydrate $(300 \mathrm{mg} / \mathrm{kg}$, IP). A 30-gauge injection needle was then stereotaxically lowered into the $\mathrm{MPFC}$ (from the bregma, AP $3.0 \mathrm{~mm}$, ML 0.8, and DV $4.2 \mathrm{~mm}$ ), through which ibotenic acid (Sigma, $50 \mu \mathrm{mol} / \mathrm{L}$, dissolved in saline) or the vehicle was infused with a micropump at the rate of $200 \mathrm{nl} /$ minute for 2 minutes. Recordings were conducted 4 to 14 days after the surgery. In all animals studied, lesions were observed in the infralimbic and ventral prelimbic division of the PFC (Figure 1). All surgical procedures were carried out according to the Guide for the Care and Use of Laboratory Animals by the U.S. Public Health Service and were approved by the Yale Animal Care and Use Committee.

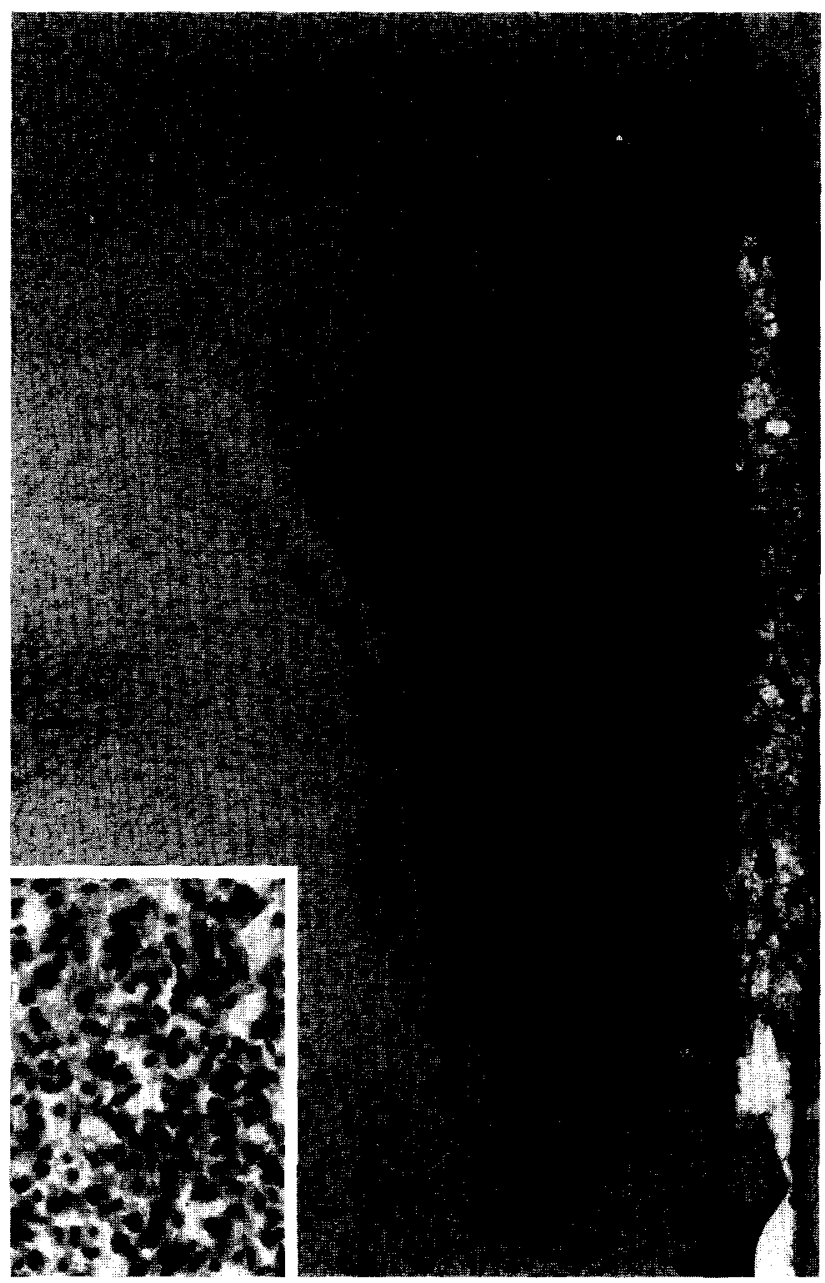

DA neurons were recorded as described previously (Bunney et al. 1973). Briefly, rats were anesthetized with chloral hydrate $(400 \mathrm{mg} / \mathrm{kg}$, IP with supplemental doses administered via the lateral tail vein) and mounted in a stereotaxic instrument. Body temperature was maintained at 36 to $37^{\circ} \mathrm{C}$ with a heating pad. Single barrel glass electrodes filled with $1.0 \mathrm{~mol} / \mathrm{L} \mathrm{NaCl}$ and $2 \%$ pontamine sky blue dye ( 5 to $15 \mathrm{M} \Omega$ ) were lowered through 12 recording tracks in a stereotaxically defined tissue block within the SN (from the lambda, AP 2.7 to $3.3 \mathrm{~mm}$, ML 1.8 to $2.2 \mathrm{~mm}$, DV 6.0 to $8.0 \mathrm{~mm}$ ) and the VTA (from the lambda, AP 2.7 to $3.3 \mathrm{~mm}$, ML 0.5 to 0.9 $\mathrm{mm}$, DV 6.0 to $8.0 \mathrm{~mm}$ ), using a hydraulic microdriver (Bunney and Grace 1978). Spontaneously active DA neurons were identified based on previously established criteria (Bunney et al. 1973; Grace and Bunney 1983). Interspike intervals were collected on-line by a PC computer (486 interfaced via a Lab-PC ${ }^{+}$, National Instrument) using programs written by one of the au-

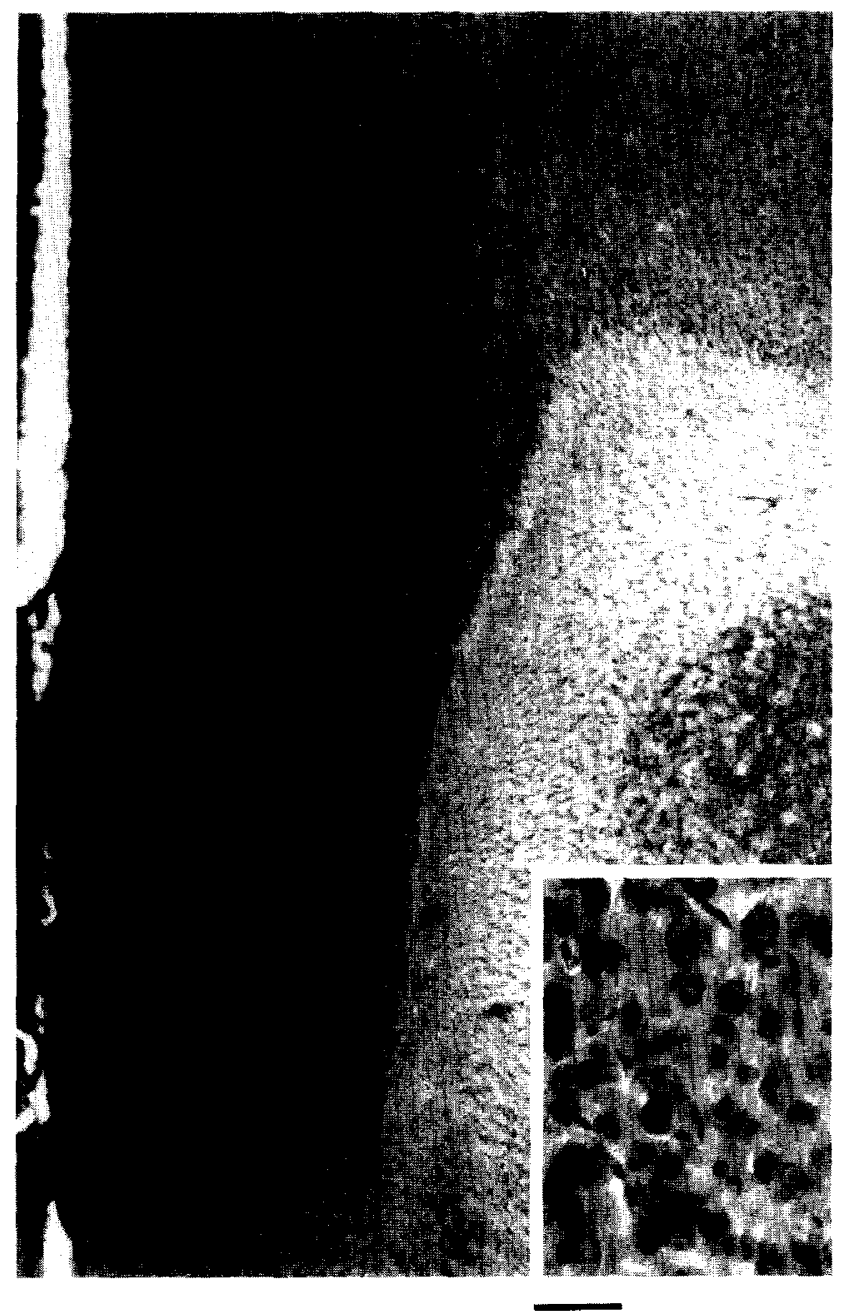

Figure 1. Photomicrographs of Cresyl violet-stained sections through the medial prefrontal cortex with ibotenic acid lesion (left, 7 days after the injection) and without (right). Inserts are cells in layer V and VI viewed under a high magnification. Note the gliosis with concomitant neuronal loss in the lesioned medial prefrontal cortex. Scale bar $=400 \mu \mathrm{m}$ and $50 \mu \mathrm{m}$ for the low and high power, respectively. 
thors (W.S.). Off-line data analysis was performed in Microsoft Excel. The onset and offset of burst firing were defined as the interspike intervals of less than 80 milliseconds and greater than 160 milliseconds, respectively (Grace and Bunney 1984). Lesion and recording sites were confirmed in Cresyl violet-stained sections. All values are expressed as mean \pm SEM. Differences between groups were evaluated by using ANOVA followed by post-hoc Tukey HSD tests (SYSTAT, Evanston, IL).

\section{RESULTS}

The activity of DA neurons was not altered by the sham operation as there was no difference in any of the parameters measured (number of active cells, firing rate or spikes in burst) between nonlesioned and sham-lesioned animals. However, DA neurons in both the VTA and $\mathrm{SN}$ were affected by the mPFC lesion (Figure 2). In the VTA, the number of active DA neurons was significantly reduced in PFC lesioned rats when compared with either nonoperated or sham-lesioned rats (nonlesion: $1.36 \pm 0.09$ cells $/$ tract, $n=10$; sham lesion: $1.40 \pm$ $0.14, n=4$; lesion: $0.56 \pm 0.11, n=7$ ). The average firing rate of the cells was, however, not significantly different between groups (nonlesion: $3.64 \pm 0.14$ spikes/second, $n=169$, sham lesion: $3.91 \pm 0.26, n=47$; lesion: $4.21 \pm$ $0.30, n=37)$. VTA DA neurons in PFC-lesioned rats also showed more bursting activity. However, the change was statistically not significant (nonlesion: $26.75 \pm 2.00 \%$ of spikes in burst, $n=169$; sham lesion: $22.90 \pm 3.70 \%$, $n=47$; lesion: $34.83 \pm 4.17 \%, n=37$ ).

Unlike in the VTA, the number of active DA cells in the SN was not significantly altered by PFC lesioning, although there was a tendency toward a decrease in the lesioned group (nonlesion: $1.15 \pm 0.1, n=6$; sham lesion: $1.02 \pm 0.11, n=5$; lesion: $0.83 \pm 1.1, n=5$ ). The basal firing rate, however, was significantly increased in PFC lesioned rats ( $4.89 \pm 0.26$ spikes/second, $n=46)$ when compared with either nonlesioned $(3.85 \pm 0.21$, $n=73)$ or sham-lesioned rats $(3.71 \pm 0.25, n=50)$. As observed in the VTA, a statistically nonsignificant increase in bursting activity was observed in the SN (nonlesion: $15.75 \pm 2.60 \%$ of spikes in burst, $n=73$; sham lesion: $15.49 \pm 3.15 \%, n=50$; lesion: $20.11 \pm 3.28 \%$, $n=46)$.

\section{DISCUSSION}

These results are consistent with the hypothesis that PFC lesions alter the activity of DA neurons located in the midbrain. The data suggest, however, that DA neurons in the VTA and the SN respond in different ways to the lesion. In the VTA, the overall activity of DA neurons was decreased, whereas that in the SN was increased by the same lesions in the PFC.

A decrease in VTA DA neuron activity in PFC-lesioned rats is consistent with previous studies showing that the PFC has an excitatory effect on DA neurons.
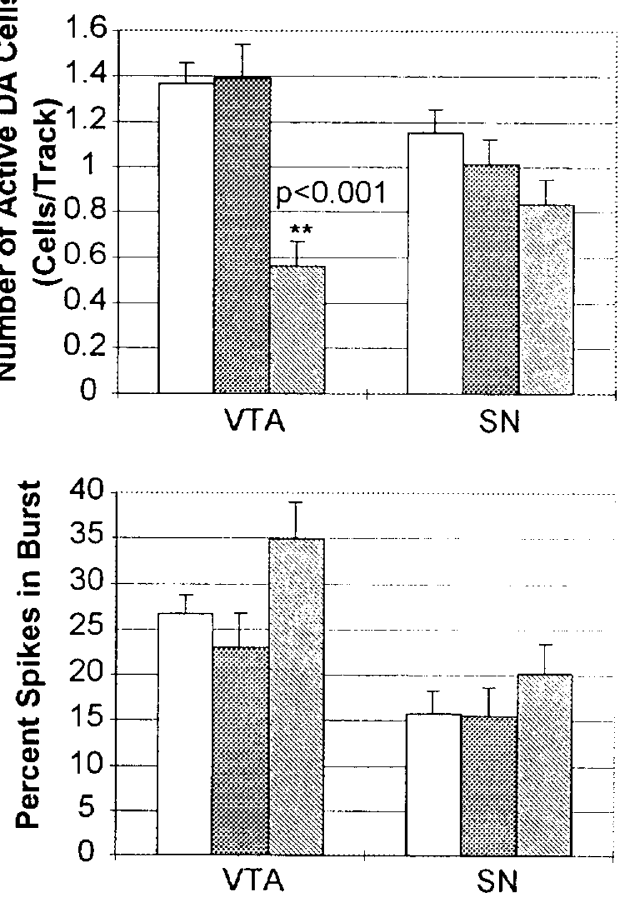
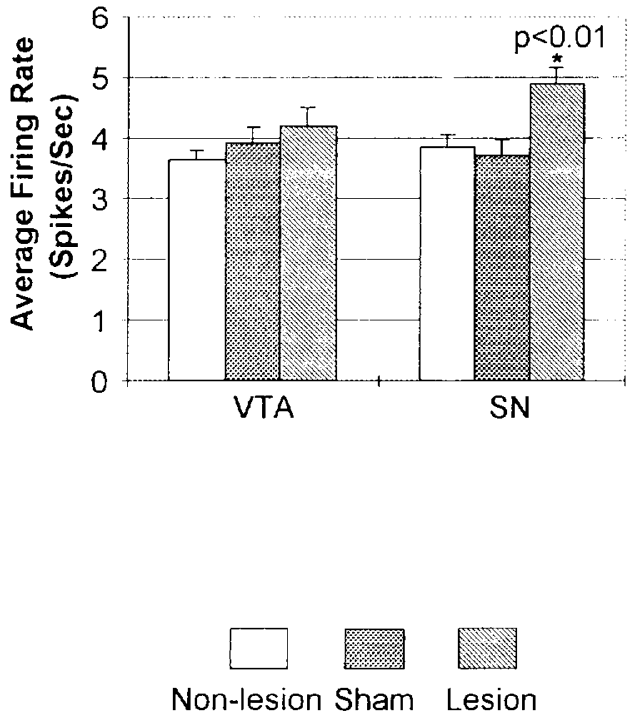

Figure 2. Effects of lesion of the mPFC on the activity of midbrain DA neurons. Upper left: The number of active DA cells in the VTA was significantly decreased in $\mathrm{mPFC}$-lesioned rats. The same lesion, however, had no effect in the SN. Upper right: $\mathrm{mPFC}$ lesion significantly increased the firing rate of SN DA neurons but had no effect on VTA DA neuron activity. Lower left: A tendency toward an increased bursting was observed for both VTA and SN DA neurons in mPFC-lesioned rats; however, the change was statistically not significant. 
Electrical and pharmacologic stimulation of the $\mathrm{mPFC}$ has been shown to increase the burst firing of spontaneously active DA neurons (Thierry et al. 1979; Gariano and Groves 1988; Murase et al. 1993), whereas cooling the PFC decreases burst firing (Svensson and Tung 1989). Recently, Taber et al. (1995) showed that application of glutamate antagonists to the VTA blocks PFC stimulation-induced DA release in the nucleus accumbens. The present study, showing a reduction in the number of spontaneously active VTA DA cells in PFClesioned rats, suggests that a tonic input from the mPFC may be necessary for some VTA DA neurons to fire spontaneously.

However, in addition to the direct PFC-VTA pathway, the PFC may affect DA neurons indirectly through other areas such as the nucleus accumbens, which receives massive input from the PFC (Sesack et al. 1989) and, in turn, projects to the VTA. Because accumbens projections to the VTA are GABAergic, PFC lesions may, via PFC-accumbens-VTA pathway, disinhibit VTA DA neurons leading to a depolarization inactivation of VTA DA neurons. Another possible explanation for our findings is that ibotenic acid injection in the PFC caused a degeneration of VTA DA neurons projecting to the PFC, resulting in a reduced number of active VTA DA neurons. Further investigation will be required to determine whether and how each of these possible mechanisms may contribute to the observed effect of PFC lesion on VTA DA neurons.

A direct PFC projection to the $\mathrm{SN}$ may also exist (Christie et al. 1985; Fiedler and Bustos 1991; Goswell and Sedgwick 1973; Kornhuber et al. 1984; Naito and Kita 1994). However, this projection seems unlikely to be responsible for the observed increase in SN DA neuron activity in PFC-lesioned rats. PFC projection neurons are believed to be excitatory, using either glutamate or aspartate. By lesioning this PFC-SN excitatory pathway, one would expect, instead of an increase, a decrease in DA neuron activity in the SN. Thus, other non-DA neurons that are affected by the PFC may be involved. One possibility is that PFC terminals synapse on primarily SN non-DA neurons (GABAergic), which, in turn, inhibit DA neurons (Fujimoto and Kita 1992). Removal of the PFC afferent may thus lead to a decrease in the activity of these inhibitory neurons and indirectly cause an increase in SN DA cell firing. The corticostriatal pathway may also play a role, as PFC lesions may decrease the activity of striatal neurons involved in the inhibitory feedback pathway of SN DA neurons.

Whereas the exact mechanism through which midbrain DA neurons are affected by PFC lesions remains to be determined, changes in the activity of DA neurons would predict corresponding changes in DA terminal release. However, variable effects of PFC lesions on DA levels have been reported. For example, Pasinetti et al. (1991) noticed a significant decrease in DA levels in the striatum after unilateral ablation of the frontal cortex. Jaskiw et al. (1990a), however, reported a transient increase in striatal DA levels. In the nucleus accumbens, PFC lesion produced either no effect or a transient increase in DA levels (Christie et al. 1986; Jaskiw et al. $1990 \mathrm{~b}$ ). When the animal is subjected to mild stress, a prolonged increase in DA release in the nucleus accumbens is observed in PFC-lesioned rats (Jaskiw et al. 1990b). Taken together, these observations suggest that a PFC lesion may affect DA neurons in different ways under different circumstances. As the experiments of the present study were performed in anesthetized animals, whether and how PFC lesions affect the activity of DA neurons in awake animals, particularly under stress, remains to be determined.

\section{ACKNOWLEDGMENTS}

This work was supported by PHS award MH28849, the Scottish Rite Schizophrenia Research Program, the National Parkinson's Foundation, the Stanley Foundation for Reseanch on the Mentally Ill, and the state of Connecticut.

\section{REFERENCES}

Bouyer JJ, Park DH, Joh TH, Pickel VM (1984): Chemical and structural analysis of the relation between cortical inputs and tyrosine hydroxylase-containing terminals in rat neostriatum. Brain Res 302:267-276

Bunney BS, Grace AA (1978): Acute and chronic haloperidol treatment: Comparison of effects on nigral dopaminergic cell activity. Life Sci 23:1715-1728

Bunney BS, Walters JR, Roth RH, Aghajanian GK (1973): Dopaminergic neurons: Effect of antipsychotic drugs and amphetamine on single cell activity. J Pharmacol Exp Ther 185:560-571

Christie MJ, Bridge S, James LB, Beart PM (1985): Excitotoxin lesions suggest an aspartatergic projection from rat medial prefrontal cortex to ventral tegmental area. Brain Res 333:169-172

Christie MJ, Rowe PJ, Beart PM (1986): Effect of exictotoxin lesions in the medial prefrontal cortex on cortical and subcortical catecholamine turnover in the rat. J Neurochem 47:1593-1597

Deutch AY (1992): The regulation of subcortical dopamine systems by the prefrontal cortex: Interactions of central dopamine systems and the pathogenesis of schizophrenia. J Neural Transm 36:61-89

Fiedler J, Bustos G (1991): Cortical ablation reduces veratridine evoked release of endogenous glutamate from superfused substantia nigra slices. Neurosci Lett 122:96-98

Fujimoto K, Kita H (1992): Responses of rat substantia nigra pars reticulata units to cortical stimulation. Neurosci Lett 142:105-109

Gariano RF, Groves PM (1988): Burst firing induced in midbrain dopamine neurons by stimulation of the medial 
prefrontal and anterior cingulate cortices. Brain Res 462:194-198

Goswell MJ, Sedgwick EM (1973): Is there a cortico-nigral tract? Some neurophysiological observations. Brain Res 50:437-440

Grace AA (1991): Phasic versus tonic dopamine release and the modulation of dopamine system responsivity: A hypothesis for the etiology of schizophrenia. Neuroscience $41: 1-2$

Grace AA, Bunney BS (1983): Intracellular and extracellular electrophysiology of nigral dopamine neurons. 1. Identification and characterization. Neuroscience 10:301-315

Grace AA, Bunney BS (1984): The control of firing pattern in nigral dopamine neurons: Burst firing. J. Neuroscience $4: 2877-2890$

Jaskiw GE, Karoun F, Freed WJ, Phillips I, Kleinman JE, Weinberger DR (1990a): Effect of ibotenic acid lesions of the medial prefrontal cortex on amphetamine-induced locomotion and regional brain catecholamine concentrations in the rat. Brain Res 534:263-272

Jaskiw GE, Karoun FK, Weinberger DR (1990b): Persistent elevations in dopamine and its metabolites in the nucleus accumbens after mild subchronic stress in rats with ibotenic acid lesions of the medial prefrontal cortex. Brain Res 534;321-323

Jin S, Fredholm BB (1994): Role of NMDA, AMPA and kainate receptors in mediating glutamate- and 4-AP-induced dopamine and acetylcholine release from rat striatal slices. Neuropharmacology 33(9):1039-1048

Kornhuber J, Kimm J-S, Kornhuber ME, Kornhuber $\mathrm{HH}$ (1984): The cortico-nigral projection: Reduced glutamate content in the substantia nigra following frontal cortex ablation in the rat. Brain Res 322:124-126

Marien M, Brien J, Jhamandas K (1983): Regional release of [3H]dopamine from rat brain in vitro: Effects of opioids on release induced by potassium, nicotine, and I-glutamic acid. Can J Physiol Pharmacol 61:43-60

Murase S, Grenhoff J, Chouver G, Gonon FG, Svensson TH (1993): Prefrontal cortex regulates burst firing and trans- mitter release in rat mesolimbic dopamine neurons studied in vivo. Neurosci Lett 157:53-56

Naito A, Kita H (1994): The cortico-nigral projection in the rat: An anterograde tracing study with biotinylated dextran amine. Brain Res 637:317-322

Ohta K, Araki N, Shibata M, Komatsumoto S, Shimazu K, Fukuuchi Y (1994): Presynaptic ionotropic glutamate receptors modulate in vivo release and metabolism of straital dopamine, noradrenaline, and 5-hydroxytryptamine: Involvement of both NMDA and AMPA/kainate subtypes. Neurosci Res 21(1):83-89

Pasinetti GM, Kohama S, Reinhard Jr JF, Cheng HW, McNeill TH, Finch CE (1991): Striatal responses to decortication. I. Dopaminergic and astrocytic activities. Brain Res. $567: 253-259$

Sesack SR, Pickel VM (1992): Prefrontal cortical efferents in the rat synapse on unlabeled neuronal targets of catecholamine terminals in the nucleus accumbens septi and on dopamine neurons in the ventral tegmental area. J Comp Neurol 320:145-160

Sesack SR, Deutch AY, Roth RH, Bunney BS (1989): Topographical organization of the efferent projections of the medial prefrontal cortex in the rat: An anterograde tracttracing study with phaseolus vulgaris leucoagglutinin. J Comp Neurol 290:213-242

Svensson TH, Tung C-S (1989): Local cooling of pre-frontal cortex induces pacemaker-like firing of dopamine neurons in rat ventral tegmental area in vivo. Acta Physiol Scand 136:135-136

Taber MT, Das S, Fibiger HC (1995): Cortical regulation of subcortical dopamine release: Mediation via the ventral tegmental area. J Neurochem 65(3):1407-1410

Thierry AM, Deniau JM, Feger J (1979): Effects of stimulation of the frontal cortex on identified output VMT cells in the rat. Neurosci Lett 15:103-107

Weinberger DR (1987): Implications for normal brain development for the pathogenesis of schizophrenia. Arch Gen Psychiatry 44:660-669 